\title{
Dengue virus sero-cross-reactivity drives antibody- dependent enhancement of infection with zika virus
}

\author{
Wanwisa Dejnirattisai ${ }^{1}$, Piyada Supasa ${ }^{1-3}$, Wiyada Wongwiwat ${ }^{1}$, Alexander Rouvinski ${ }^{4,5}$, \\ Giovanna Barba-Spaeth $^{4,5}$, Thaneeya Duangchinda ${ }^{6}$, Anavaj Sakuntabhai ${ }^{7,8}$, Van-Mai Cao-Lormeau ${ }^{9}$, \\ Prida Malasit ${ }^{2,6}$, Felix A Rey ${ }^{4,5}$, Juthathip Mongkolsapaya ${ }^{1,2} \&$ Gavin R Screaton $^{1}$
}

\begin{abstract}
Zika virus (ZIKV) was discovered in 1947 and was thought to lead to relatively mild disease. The recent explosive outbreak of ZIKV in South America has led to widespread concern, with reports of neurological sequelae ranging from Guillain Barré syndrome to microcephaly. ZIKV infection has occurred in areas previously exposed to dengue virus (DENV), a flavivirus closely related to ZIKV. Here we investigated the serological cross-reaction between the two viruses. Plasma immune to DENV showed substantial cross-reaction to ZIKV and was able to drive antibody-dependent enhancement (ADE) of ZIKV infection. Using a panel of human monoclonal antibodies (mAbs) to DENV, we showed that most antibodies that reacted to DENV envelope protein also reacted to ZIKV. Antibodies to linear epitopes, including the immunodominant fusion-loop epitope, were able to bind ZIKV but were unable to neutralize the virus and instead promoted ADE. Our data indicate that immunity to DENV might drive greater ZIKV replication and have clear implications for disease pathogenesis and future vaccine programs for ZIKV and DENV.
\end{abstract}

ZIKV is an arbovirus that belongs to the family flaviviridae and is transmitted to man by Aedes mosquitos ${ }^{1}$. ZIKV was first isolated from a sentinel rhesus monkey in the Zika forest of Uganda in 1947 and has subsequently been found in mosquitos and humans ${ }^{1}$. Until recently, ZIKV was not viewed as a particularly important pathogen, as the majority of infections are asymptomatic ${ }^{2}$. Symptomatic cases of ZIKV resemble mild cases of dengue fever, with fever, myalgia, arthralgia, headache, conjunctivitis and $\mathrm{rash}^{3}$.

Until recently, cases were sporadic, largely in Africa and Southeast Asia, and epidemic activity had not been observed ${ }^{1}$. A large outbreak of ZIKV occurred on Yap island in the Western Pacific in 2007, then spread through Oceania and reached Brazil in 2015, where it rapidly spread to other South American countries ${ }^{1,4-7}$.

It is now apparent that ZIKV infection can cause substantial neurological complications; an increase in cases of Guillain Barré syndrome was first reported following the outbreak in French Polynesia in 2013 (ref. 8). Dramatic increases in the incidence of microcephaly originating in northeastern Brazil were reported in late 2015, coincident with a large increase in ZIKV infection ${ }^{9,10}$. These increases in Guillain Barré syndrome and microcephaly led the World Health Organization to declare ZIKV a public health emergency in February 2016 (ref. 11).

ZIKV can be carried by a variety of Aedes mosquitos, but the principal species responsible for the current outbreaks is thought to be Aedes aegypti1,3. In parts of Brazil, A. aegypti is also spreading DENV and chikungunya viruses concurrently with ZIKV ${ }^{12-17}$. In the past 20 years, DENV has spread through areas of South America, and the seroprevalence of DENV in some areas affected by ZIKV exceeds $90 \%$ (refs. 18-20).

DENV exists as four serotypes that differ by $30-35 \%$, and the DENV serocomplex in turn differs from ZIKV by $41-46 \%$ (in amino acid sequence of the envelope protein $)^{21}$. Published reports have shown difficulty in distinguishing DENV infection versus ZIKV infection serologically, indicative of a degree of antigenic similarity between the viruses $^{4,22,23}$.

Following a primary DENV infection, a person develops life-long immunity to the infecting serotype but not to the other serotypes ${ }^{24,25}$. In DENV-endemic areas, all four serotypes of DENV frequently circulate together or cyclically replace each other, which means that multiple sequential infections are common ${ }^{26}$. One of the interesting features of DENV infection is that the life-threatening complications that lead to dengue hemorrhagic fever are more common after secondary infection than after primary infection ${ }^{21}$. One theory to explain this is $\mathrm{ADE}^{21}$. The $\mathrm{ADE}$ hypothesis suggests that antibodies generated during a primary infection with DENV will not be of sufficient concentration or avidity to neutralize a secondary infection with DENV of a different serotype that differs in amino acid sequence by $30-35 \%$. However, they might

${ }^{1}$ Division of Immunology and Inflammation, Department of Medicine, Hammersmith Campus, Imperial College London, UK. ${ }^{2}$ Dengue Hemorrhagic Fever Research Unit, Office for Research and Development, Siriraj Hospital, Faculty of Medicine, Mahidol University, Bangkok, Thailand. ${ }^{3}$ Graduate Program in Immunology, Department of Immunology, Faculty of Medicine, Siriraj Hospital, Mahidol University, Bangkok, Thailand. ${ }^{4}$ Institut Pasteur, Département de Virologie, Unité de Virologie Structurale, Paris, France. ${ }^{5}$ CNRS UMR 3569 Virologie, Paris, France. ${ }^{6}$ Medical Biotechnology Unit, National Center for Genetic Engineering and Biotechnology, National Science and Technology Development Agency, Pathumthani, Thailand. 7 Institut Pasteur, Functional Genetics of Infectious Diseases Unit, Paris, France. ${ }^{8}$ CNRS URA3012, Paris, France. ${ }^{9}$ Unit of Emerging Infectious Diseases, Institut Louis Malardé, Papeete, Tahiti, French Polynesia. Correspondence should be addressed to G.R.S. (g.screaton@imperial.ac.uk) or J.M. (j.mongkolsapaya@imperial.ac.uk). 
Table 1 Study subjects

\begin{tabular}{lllll}
\hline Patient identifier & Age (years) & Sex & Severity & Infection serotype \\
\hline K01 & 5 & $\mathrm{M}$ & DHF1 & DENV 1 \\
K02 & 2 & $\mathrm{~F}$ & DHF3 & DENV 1 \\
K03 & 15 & $\mathrm{M}$ & DHF1 & DENV 1 \\
K04 & 5 & $\mathrm{M}$ & DF & DENV 1 \\
K06 & 10 & $\mathrm{~F}$ & DHF1 & DENV 2 \\
K07 & 11 & $\mathrm{M}$ & DHF3 & DENV 2 \\
K08 & 11 & $\mathrm{~F}$ & DHF1 & DENV 2 \\
K09 & 14 & $\mathrm{~F}$ & DHF1 & DENV 2 \\
K11 & 14 & $\mathrm{~F}$ & DHF2 & DENV 3 \\
K12 & 14 & $\mathrm{M}$ & DHF1 & DENV 3 \\
K13 & 11 & $\mathrm{~F}$ & DHF2 & DENV 3 \\
K14 & 13 & $\mathrm{M}$ & DF & DENV 3 \\
K15 & 15 & $\mathrm{~F}$ & DHF2 & DENV 4 \\
K16 & 5 & $\mathrm{M}$ & DF & DENV 4 \\
K17 & 8 & $\mathrm{~F}$ & DF & DENV 4 \\
K18 & 6 & $\mathrm{M}$ & DF & DENV 4 \\
\hline
\end{tabular}

Information on DENV-infected patients enrolled in the study. Severity is assigned grades of dengue hemorrhagic fever (DHF1-DHF3) or dengue fever (DF). M, male; $\mathrm{F}$, female.

still opsonize the secondary virus and target it for Fc-receptor-mediated endocytosis into myeloid cells such as monocytes and macrophages (which are the principal site of DENV replication) and thus drive higher viral loads. ADE can be readily demonstrated in vitro and has also be shown to drive higher viral loads of DENV in animal models ${ }^{27-30}$.

Here we took advantage of a panel of human mAbs generated from DENV-infected subjects to demonstrate substantial cross-reactivity between DENV and ZIKV. Most mAbs to DENV also bound to ZIKV, but those that recognize the major linear fusion-loop epitope (FLE) did not neutralize ZIKV, whereas they showed neutralizing activity against DENV. DENV-immune plasma and mAbs to DENV potently enhanced ZIKV infection, which suggests the possibility that preexisting immunity to DENV might increase ZIKV replication.

\section{RESULTS}

\section{DENV plasma cross-reacts with ZIKV}

We studied a cohort previously established to investigate DENV infection in children in Khon Kaen in northeast Thailand. After informed consent was obtained, subjects were enrolled in the study; plasma and peripheral blood mononuclear cells were collected during their hospital stay for the acute phase of the illness, and samples were also collected during the convalescent phase, around 6 months following discharge from the hospital. Samples were collected between 2002 and 2004 (Table 1); at this time, ZIKV was not described in the Khon Kaen region. Plasma collected from subjects around 6 months following secondary infection with DENV serotypes 1-4 was assessed for its binding to ZIKV and DENV by capture ELISA. In all cases, DENV-immune plasma bound to both DENV and ZIKV (Fig. 1). The overall patterns of binding to ZIKV strains originating in Africa (HD78788) or French Polynesia (PF13) were similar (Fig. 1).

Next we assessed neutralization of ZIKV by plasma collected from DENV-infected subjects during the convalescent phase. All such plasma was able neutralize DENV nearly $100 \%$ at the lowest dilution used (1:50) (Fig. 2a). However, neutralization of ZIKV was considerably less efficient, with most plasma samples showing no appreciable neutralization at a dilution of 1:50 (Fig. 2b). The three samples that showed appreciable neutralization of ZIKV HD78788 were among the strongest neutralizers of DENV (Fig. 2b). The finding that plasma containing antibodies to DENV substantially cross-reacted with ZIKV prompted us to determine whether it was able to promote ADE.

\section{DENV plasma potently induces ADE}

We assessed the ability of plasma from DENV-infected subjects to promote ADE in the human myeloid cell line U937, which is relatively resistant to infection by DENV in the absence of $\mathrm{ADE}^{31}$. Here, we found that U937 cells were also poorly permissive to ZIKV, with infection of $<0.6 \%$ of cells in the absence of ADE (Fig. 3a). ZIKV was pre-incubated with a titration of pooled serum collected from convalescent subjects at 1-2 weeks after recovery from infection with DENV and was then used to infect U937 cells. Pooled serum from convalescent subjects led to substantial enhancement ( $>100$-fold) of infection of cells by ZIKV strains PF13 and H78788 (Fig. 3b). As expected, plasma pooled from control subjects not infected with DENV did not enhance such infection (Fig. 3b). Next we assessed a panel of convalescent plasma collected from subjects around 6 months following acute secondary infection with DENV. In all but one case, plasma from DENV-infected subjects enhanced the infection of cells with ZIKV, with a median increase of 12 -fold for infection by the HD78788 strain of ZIKV (Fig. 3c,d). In summary, these results demonstrated that cross-reacting antibodies to $\mathrm{DENV}$ were able promote $\mathrm{ADE}$ of ZIKV infection but were poorly neutralizing.

\section{Cross-reaction of mAbs to DENV}

We used a previously created a panel of human mAbs that react to the DENV envelope protein, generated from plasmablasts isolated from DENV-infected patients ${ }^{27}$. Detailed epitope mapping of these antibodies has demonstrated three broad reactivities. Around one third of the antibodies react to the well-described fusion-loop epitope (FLE), and another one third have not been definitively mapped but, like the a

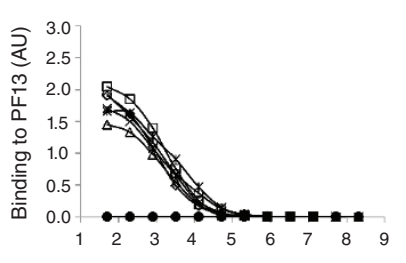

Plasma dilution $\left(\log _{10}\right.$ reciprocal value)

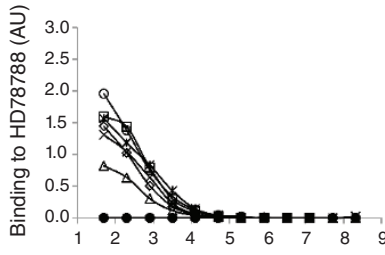

Plasma dilution $\left(\log _{10}\right.$ reciprocal value)

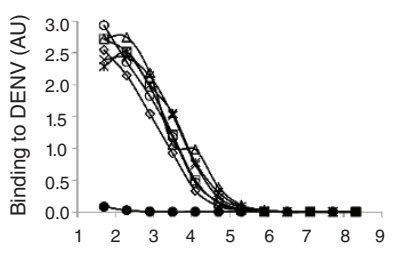

Plasma dilution ( $\log _{10}$ reciprocal value) b

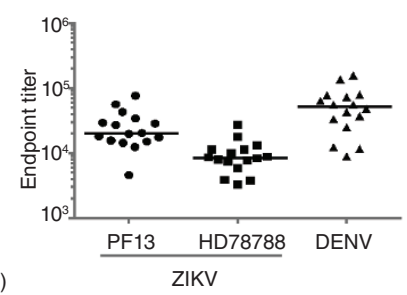

Figure 1 DENV-immune plasma cross-reacts with ZIKV. (a) Capture ELISA of the binding of plasma from DENV-infected subjects collected at 6 months after discharge from the hospital (six samples (open symbols or ' $x$ ')) and plasma pooled from control subjects not infected with DENV (filled circles) to ZIKV strains PF13 and HD78788 and DENV, assayed at 12 concentrations of plasma and presented (in arbitrary units (AU)) as titration curves; for DENV, the DENV serotype corresponding to the previous acute infection was used as the capture ELISA antigen. (b) Capture ELISA of the endpoint titers of DENVimmune plasma ( $n=16$ samples) against ZIKV (strains PF13 and HD78788) and DENV. Each symbol represents an individual sample; small horizontal lines indicate the median. Data are representative of one experiment with 16 samples. 
a

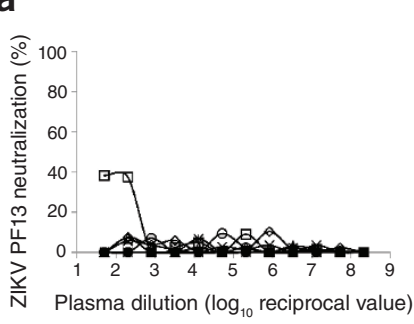

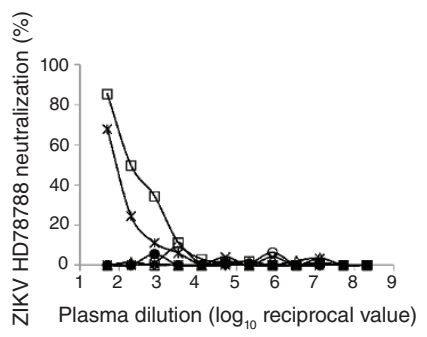

b

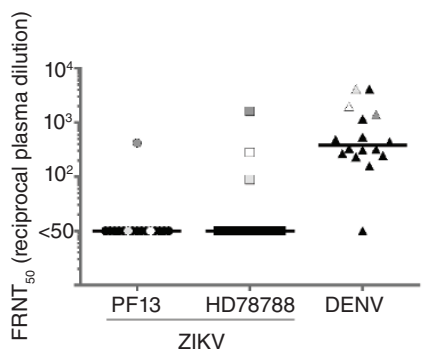

Figure 2 Neutralization of ZIKV by DENV-immune plasma. (a) Neutralization of ZIKV strains PF13 and HD78788 and DENV (on Vero cells) in the presence of plasma from DENV-infected subjects (obtained as in Fig. 1; six samples (open symbols or ' $x$ ')) or plasma pooled from non-DENV-infected subjects (negative control; filled circles), assayed at 12 different dilutions; results calculated as (foci in the absence of plasma- foci in the presence of plasma / foci in the absence of plasma) $\times 100$. (b) Neutralization of infection with ZIKV or DENV by plasma from DENV-infected subjects ( $n=16$ ), presented as the concentration that results in 50\% reduction in a focus-reduction-neutralization test (FRNT 50 ), calculated as ((foci in the absence of plasma -foci in the presence of plasma) / foci in the absence of plasma) $\times 100$. Each symbol represents an individual sample; small horizontal lines indicate the median. Data are representative of one experiment with 16 samples assayed in duplicate.

antibodies to FLE, they react to envelope protein, by immunoblot analysis (called 'non-FLE' here, as the antibodies are not sensitive to substitution of residue Trp101 of the envelope protein) ${ }^{27}$. A group of around 50 antibodies do not react to envelope protein, by immunoblot analysis, and bind only to intact viral particles ${ }^{27}$. These antibodies have been shown by cryo-electron microscopy and X-ray crystallography to bind to a conformational quaternary epitope formed at the interface of two envelope protein monomers that make up the basic head-to-tail dimer, 90 of which are arranged in icosahedral symmetry into the DENV glycoprotein shell ${ }^{27,32}$. This new epitope has been called the 'envelope dimer epitope' (EDE) and is subdivided this into two groups, EDE1 and EDE2, on the basis of sensitivity to removal of the $\mathrm{N}$-linked glycan at Asn153 in the envelope protein (the binding of mAbs to EDE2 is diminished by

Figure 3 DENV plasma enhances ZIKV infection. (a) Flow cytometry of U937 cells infected with ZIKV strain PF13 or HD78788 or DENV serotype 2 in the absence (-PCS) or presence (+PCS) of serum pooled from convalescent subjects, at a dilution of $1: 10,000$ (the dilution that results in peak enhancement). Numbers in outlined areas indicate percent cells positive (right) or negative (left) for staining with mAb 4G2 (to DENV envelope protein). SSC, side scatter. (b) Infection of U937 cells with ZIKV (strain PF13 or HD78788) or DENV serotype 2 in the presence of pooled serum from convalescent subjects (PCS) or plasma pooled from control subjects not infected with DENV (PND), presented as the ratio of viral titer in the presence of plasma to viral titer in the absence of plasma. (c) Infection of U937 cells with ZIKV (strain PF13 or HD78788) or DENV serotype 2 in the presence of serially diluted plasma from subjects infected with DENV (six samples; as in Fig. 1) or plasma pooled from control subjects not infected with DENV (negative control; filled circles), at 12 different dilutions, presented as in $\mathbf{b}$. (d) Peak enhancement of the infection of U937 cells with ZIKV or DENV via plasma from DENV-infected subjects ( $n=16$ samples), at 12 different dilutions, presented as in $\mathbf{b}$. Each symbol represents an individual sample; small horizontal lines indicate the median. Data are representative of two experiments $(\mathbf{a}, \mathbf{b})$ or one experiment with 16 samples (c,d).

removal of Asn153, while the binding of mAbs to EDE1 is not $)^{27}$. Some antibodies to EDE are fully cross-reactive to all four DENV serotypes and are able to broadly neutralize infection in the picomolar range ${ }^{27}$.

Binding of the panel of mAbs to DENV to ZIKV was assessed by capture ELISA and compared with their binding to DENV (Fig. 4a). The profile of their binding to the African (HD78788) and French Polynesian (PF13) strains of ZIKV was highly similar; all of the mAbs to FLE cross-

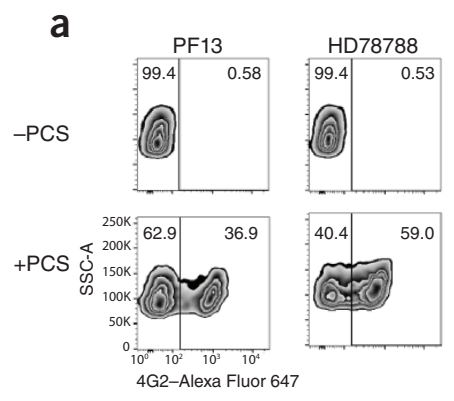

b
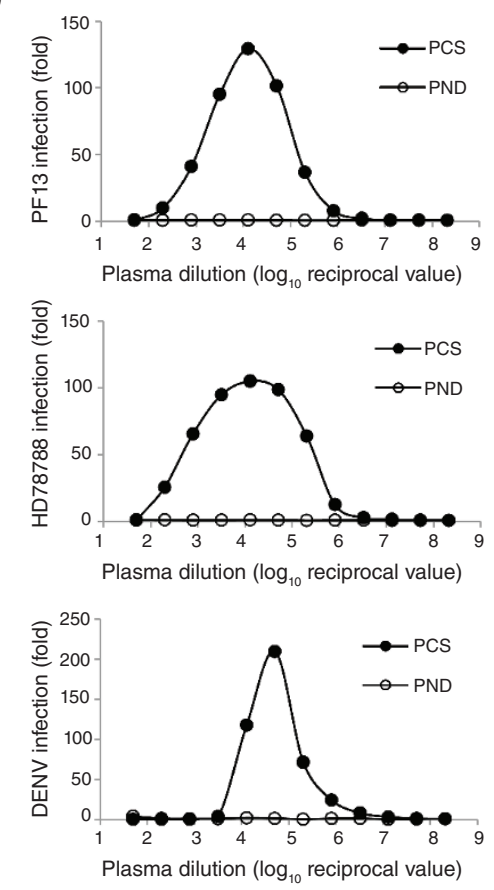
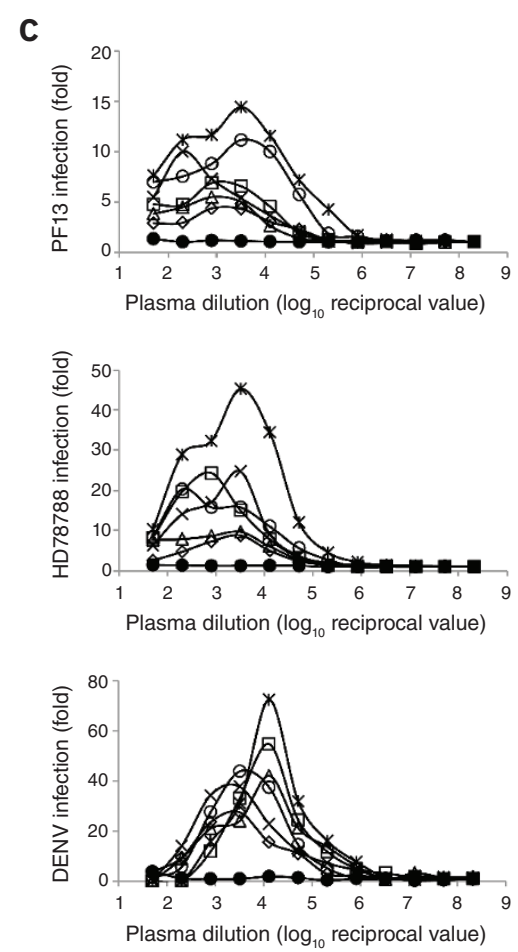

d

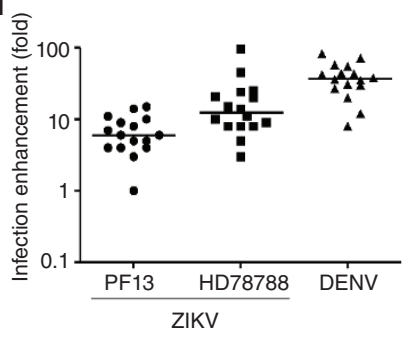


a
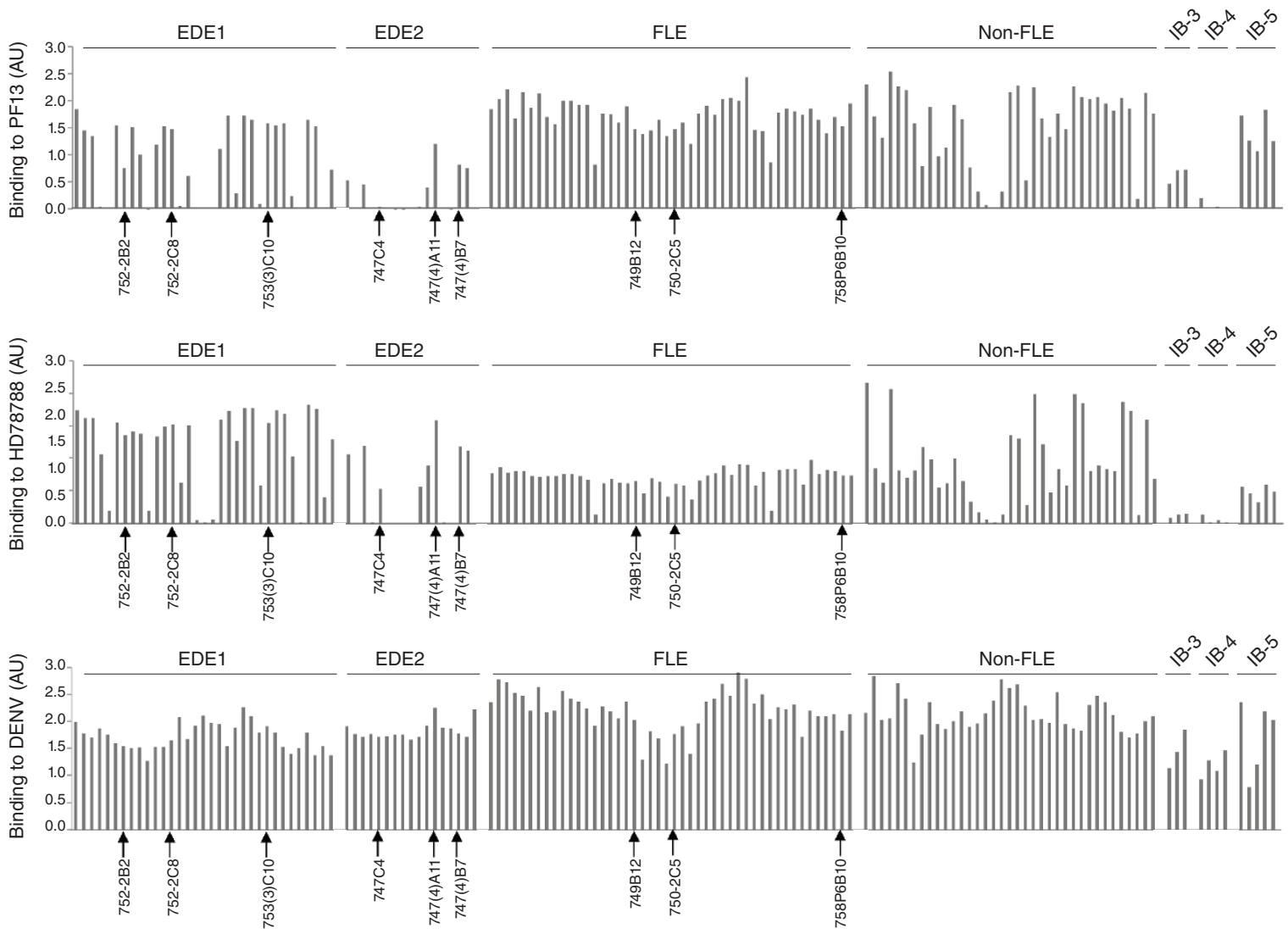

Figure 4 Human mAbs to DENV bind to ZIKV. (a) Binding of ZIKV strain PF13 or HD78788 and DENV serotype 1 by mAbs to EDE1 (33 mAbs), EDE2 (17 mAbs), FLE (46 mAbs) or non-FLE (37 $\mathrm{mAbs}$ ) (each at $10 \mu \mathrm{g} / \mathrm{ml}$; identified along horizontal axes) or by mAbs that were poorly neutralizing but reacted (by immunoblot analysis) to a conformational epitope that was lost when the envelope protein was denatured (12 mAbs: IB-3 (three mAbs), IB-4 (four $m A b s$ ) and IB-5 (five mAbs)); upward arrows indicate $\mathrm{mAbs}$ used in subsequent experiments (Figs. $\mathbf{4 b}, \mathbf{5}$ and 6). (b) Binding of ZIKV PF13 or HD78788 by various mAbs (keys) to EDE1, EDE2 and FLE (above plots; nine total: three for each epitope), presented as titration curves. Data are representative of three independent experiments (mean \pm s.e.m. in b). b
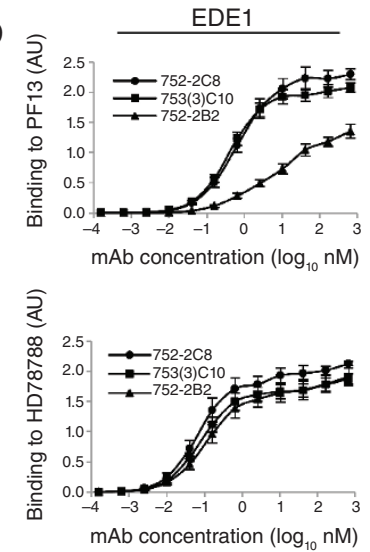
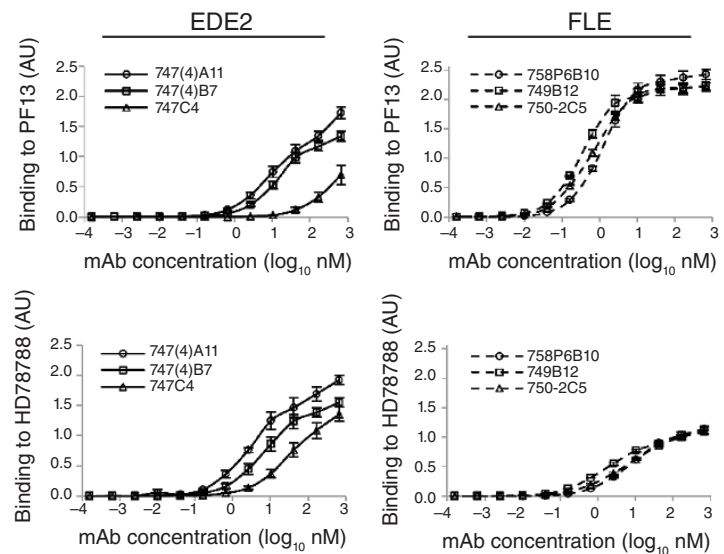

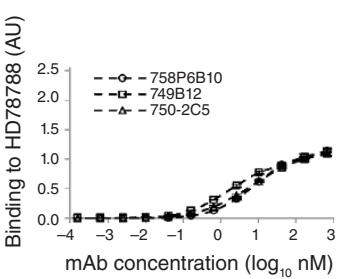

reacted with ZIKV, and 36 of 37 of mAbs to non-FLE cross-reacted with $\mathrm{ZIKV}$, whereas the cross-reaction of the mAbs to EDE1 or EDE2 was variable, with 27 of $33 \mathrm{mAbs}$ to EDE1 binding to ZIKV, and 8 of $17 \mathrm{mAbs}$ to EDE2 binding to ZIKV (Fig. 4a). Binding curves showed lower avidity of binding of ZIKV by mAbs to EDE2 versus binding by mAbs to EDE1 and lower avidity of mAb 752-2B2 (to EDE1) (Fig. 4b).

It has been demonstrated that almost all mAbs generated against DENV promote ADE, and this includes all of the human mAbs tested before $^{27}$. Because the mAbs to DENV cross-reacted with ZIKV, we next assessed the ability of mAbs to DENV to promote ADE of the infection of U937 cells by ZIKV. First, we tested three mAbs to FLE that showed no neutralization activity against $\mathrm{ZIKV}^{33}$. All of these antibodies promoted ADE, enhancing infection of the HD78788 strain by 39 - to 91-fold relative to the infection of this strain of ZIKV incubated with no antibody (Fig. 5). As expected, the ZIKV-neutralizing $\mathrm{mAb}$ to $\mathrm{EDE}^{33}$ also promoted ADE of ZIKV infection when added at sub-neutralizing concentrations, although peak enhancement was seen with lower concentrations than the concentrations of $\mathrm{mAb}$ to FLE required for peak enhancement. This demonstrated that mAbs isolated from DENVinfected patients, with different specificities, had broad cross-reactivity to ZIKV.

\section{mAbs to EDE can inhibit ADE of DENV plasma}

FLE and EDE are overlapping epitopes, as the footprint of the EDE also covers the fusion-loop region ${ }^{32}$. To investigate whether mAbs to DENV could overcome ADE induced by serum containing polyclonal antibodies to DENV, we added a titration of mAbs to DENV to ZIKV incubated with an enhancing concentration of serum containing antibodies to DENV, then assessed the infection of U937 cells. mAbs to FLE had no effect, whereas the mAbs to EDE1, except 752-2B2 (which has lower 
Figure 5 Human mAbs to DENV enhance ZIKV infection. Infection of U937 target cells by ZIKV strain PF13 or HD78788

(key) in the presence of mAbs to DENV (nine total; three each for mAbs to EDE1, EDE2 and FLE), presented as the ratio of viral titer in the presence of $m A b$ to viral titer in the absence of $\mathrm{mAb}$. Data are from three independent experiments (mean \pm s.e.m.).

avidity for ZIKV), were able to potently inhibit ADE of infection with the PF13 strain of ZIKV, with $50 \%$ inhibition occurring at titers of $0.091 \pm 0.007 \mathrm{mg} / \mathrm{ml}$ (for $\mathrm{mAb} 752-2 \mathrm{C} 8$ ) and $0.034 \pm$ $0.006 \mathrm{mg} / \mathrm{ml}$ (for $\mathrm{mAb} 753(3)$ C10) (Fig. 6). The mAbs to EDE2 of lower avidity for ZIKV than that of the mAbs to EDE1 were not able to inhibit ADE in this assay. These studies demonstrated that antibodies to EDE1 potently inhibited ADE of infection with ZIKV and that when present at sufficient concentrations, they were protective in vivo.

\section{DISCUSSION}

The recent explosion of ZIKV infection in South America, with associated Guillain Barré syndrome and microcephaly, is of great concern ${ }^{8-10}$. Guillain Barré syndrome is a relatively rare complication, estimated to affect $0.024 \%$ of ZIKV-infected people, but owing to the scale of the
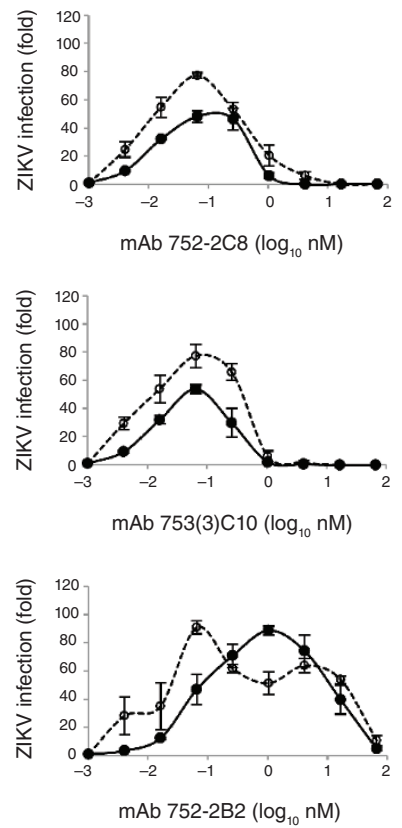

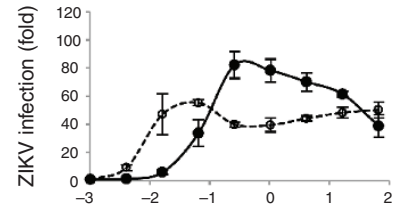

$\mathrm{mAb} 747(4) B 7\left(\log _{10} \mathrm{nM}\right)$
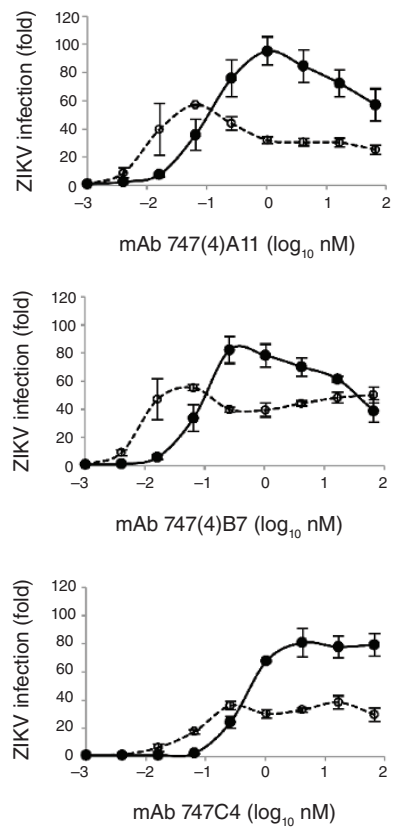

mAb 747(4)A11 $\left(\log _{10} n M\right)$
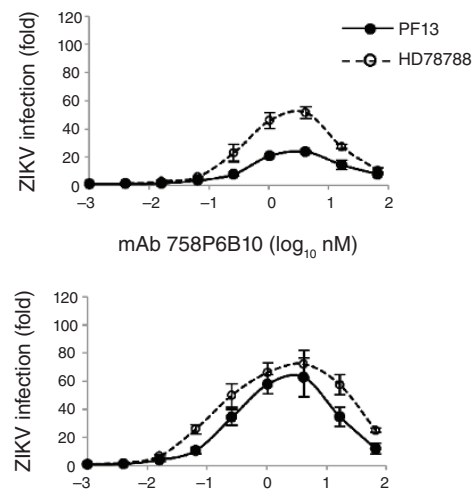

$\mathrm{mAb} 749 \mathrm{~B} 12\left(\log _{10} n M\right)$

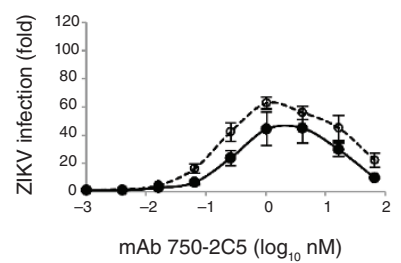

ZIKV epidemic, this still translates to large number of cases ${ }^{8}$. Much work still needs to be performed to elucidate the exact causes of the microcephaly; however, it is becoming increasingly clear that this is caused by intrauterine infection of the developing brain ${ }^{10,34-37}$. ZIKV has been shown in animal models to infect the placenta and stunt growth and is also able to cross the placenta and infect the brain ${ }^{38-40}$. Furthermore, in vitro, ZIKV can infect neural cell cultures and disrupt development in neurospheres ${ }^{41,42}$. The exact risk of neurological damage following
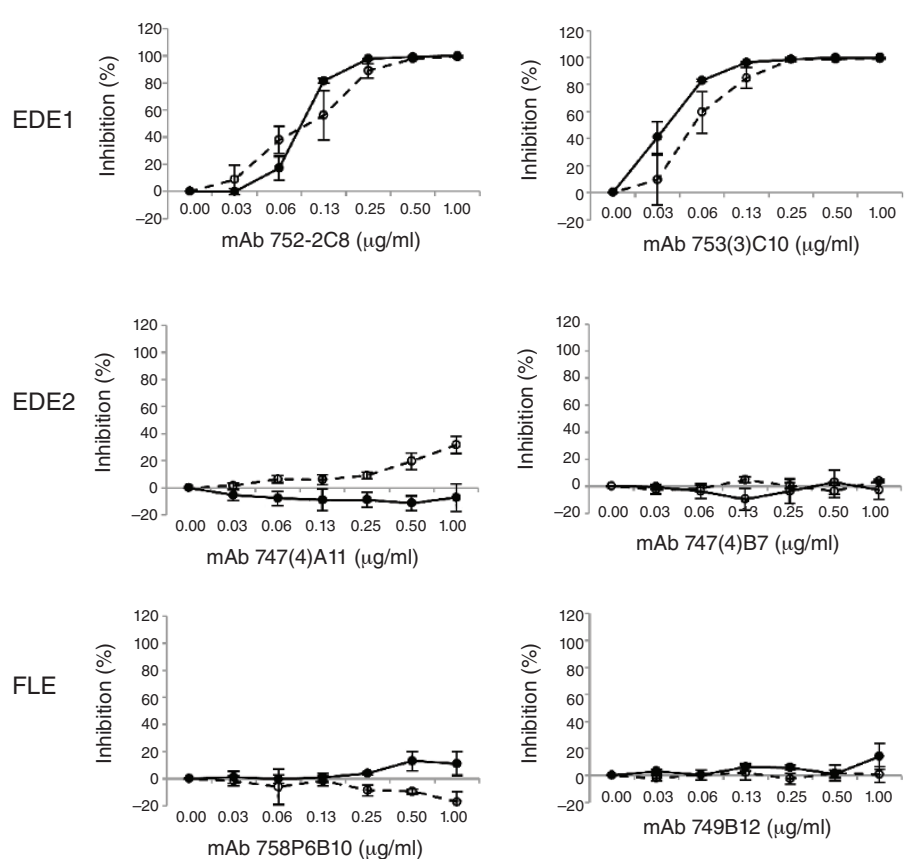
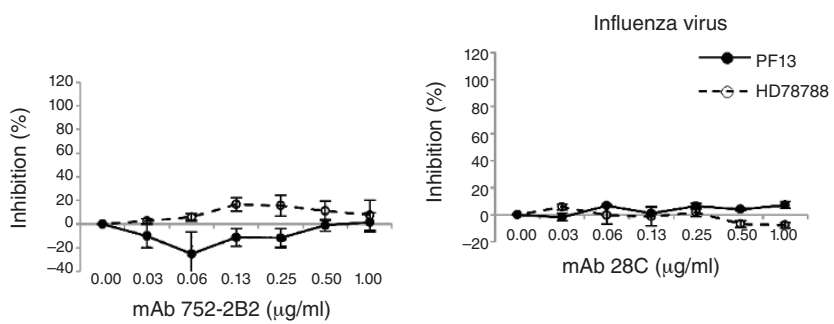
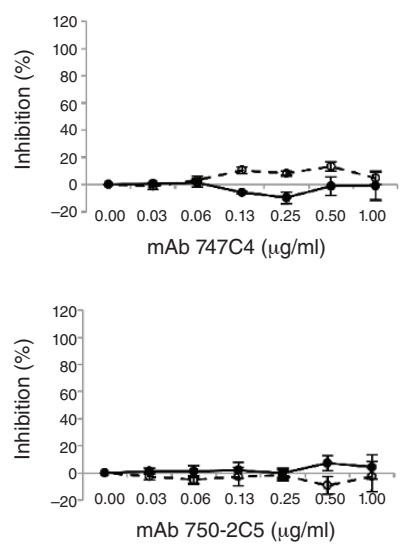

Figure 6 Human mAbs to EDE1 inhibit the ADE of serum from DENV-infected subjects. Infection of U937 cells with ZIKV strain PF13 or HD78788 (key) in the presence of pooled serum from convalescent subjects (at a dilution of 1:10,000, as in Fig. 3a) together with serially diluted mAbs to DENV (nine total; three each for mAbs to EDE1, EDE2, and FLE) or mAb 28C to influenza virus (negative control; top right), presented as (foci in the absence of mAb - foci in the presence of $\mathrm{mAb} /$ foci in the absence of $\mathrm{mAb}) \times 100$. Data are from three independent experiments (mean \pm s.e.m.). 
maternal infection remains to be determined, but early studies suggest that this risk may be up to $22 \%$ in the first trimester, although other reports from the French Polynesia put the risk at around 1\% (refs. 43,44).

ZIKV is spread by Aedes mosquitos, and in South America, these mosquitos are now also promoting epidemic spread of DENV and chikungunya viruses ${ }^{12}$. In many areas affected by ZIKV, seropositivity for DENV is very high, and in such areas there is great difficulty in distinguishing ZIKV infection versus DENV infection serologically ${ }^{19,20,23}$. In this paper, we have demonstrated substantial cross-reactivity of the antiDENV serological response toward ZIKV. Most plasma containing antibodies to DENV poorly neutralized ZIKV but potently induced ADE. However, some plasma samples from DENV-infected subjects showed appreciable neutralization of ZIKV: one sample neutralized the PF13 strain, and three samples neutralized the HD78788 strain. Although it is not formally possible to exclude the possibility of previous exposure of these subjects to ZIKV, there were no reports of ZIKV in the Khon Kaen region in 2002-2004, when the samples were collected; it is more likely they had higher circulating concentrations of cross-reactive neutralizing antibodies to DENV and ZIKV, such as those that recognize the EDE1 epitope $^{33}$. It is noteworthy that the three plasma samples that showed appreciable neutralization of ZIKV were among the four most potent DENV-neutralizing plasma samples.

A related paper has analyzed the neutralization of ZIKV by human $\mathrm{mAbs}$ to $\mathrm{DENV}^{33}$. Interestingly, antibodies to FLE, which form a major part of the antibody response in DENV infection ${ }^{21}$ and which, as shown here, promoted $\mathrm{ADE}$, failed to neutralize infection ${ }^{33}$. Antibodies that react to FLE are known to be broadly reactive across flaviviruses; however, although often strong cross-reaction is measured by ELISA, these antibodies rarely show cross-neutralizing activity, perhaps because their epitopes are poorly exposed on native virus particles ${ }^{45}$. In addition, mAbs to EDE1 show potent neutralization of ZIKV in a picomolar range similar to the range found for neutralization of DENV, while mAbs to EDE2 also neutralize ZIKV but not as potently as mAbs to EDE1 do ${ }^{27,33}$. Those results are presented together with X-ray crystallographic structures of the antigen-binding fragments of EDE1 and EDE2 in complex with the ZIKV envelope protein ${ }^{33}$. During the preparation of this manuscript, a report investigating the cross-reactivity of antibodies to DENV with ZIKV was released ${ }^{46}$. Those authors describe cross-reaction of DENV-immune serum with ZIKV and demonstrate that two human mAbs to FLE are able to enhance ZIKV infection but do not cause neutralization.

$\mathrm{ADE}$ was first recognized nearly 50 years ago in DENV infection and is believed to be one of the factors that drive increased severity of secondary infections, which is a hallmark of DENV ${ }^{29}$. The risk of ADE has made the development of vaccines against DENV particularly difficult. The most advanced vaccine against DENV, Dengvaxia (chimeric yellow fever-DENV tetravalent dengue vaccine; produced by Sanofi Pasteur) has just been licensed in several countries and gives some protection from infection; it is estimated that it will reduce the burden of disease by $10-30 \%$ over a 30 -year period if deployed in DENV-endemic countries ${ }^{47}$.

Dengvaxia is a tetravalent live attenuated vaccine in which sequences encoding the precursor membrane protein and envelope proteins that make up the glycoprotein shell of the DENV are combined with sequences encoding the non-structural proteins of the attenuated vaccine against the $17 \mathrm{D}$ strain of yellow fever virus ${ }^{21}$. Dengvaxia seems to give protection to people who have been previously infected with DENV, but its efficacy is lower when given to DENV-naive vaccinees ${ }^{21,47}$.

An analysis of the vaccine trials of Dengvaxia of longer term has raised some safety concerns ${ }^{21}$. In groups of patients under 9 years of age, hospitalization from DENV infection was greater for vaccinated children than for the non-vaccinated control group. This might represent ADE of infection in children who at entry to the study trial were DENV naive and had been primed by but not protected by the vaccine. For this reason, the vaccine is not licensed for use in children under 9 years of age and, furthermore, it is recommended that it be used only in populations in which the seroprevalence of prior DENV exposure in the age group to be vaccinated is $70 \%$ or greater ${ }^{47}$.

There is now great pressure to produce a vaccine against $\mathrm{ZIKV}$, and in this context, the extensive serological cross-reaction between DENV and ZIKV must be considered. It is likely that the vaccine will need to be deployed in areas with high seroprevalence for DENV, and raising de novo ZIKV-neutralizing responses in such a setting might be challenging. There is also the possibility that vaccination of DENV-naive subjects against ZIKV might promote ADE of DENV infection and, conversely, that vaccination against DENV might promote $\mathrm{ADE}$ of ZIKV infection.

The results described here have shown a complex serological interaction between DENV and ZIKV. The precise reason for the explosion of ZIKV infection and its complications in Brazil will need to be fully determined, but it is possible that preexisting immunity to DENV is driving greater ZIKV replication in ZIKV-infected people, which might in turn create a greater risk of complications following infection and transmission of ZIKV to mosquitoes. The possibility that ADE of infection might aid the transplacental transfer of ZIKV also needs to be investigated. The timing of DENV infection versus that of ZIKV infection might also be important, as cross-reactive protective and enhancing immunity might change over time following DENV infection.

In summary, although ZIKV differs from DENV by around $41-46 \%$ (in the sequence of the envelope protein), the similarities are sufficient to allow cross-reaction of antibodies to DENV with ZIKV and to drive ADE of infection. In this context, ZIKV could be considered a fifth member of the DENV serocomplex, a factor that must be considered in vaccine approaches to these two viruses.

\section{METHODS}

Methods and any associated references are available in the online version of the paper.

Note: Any Supplementary Information and Source Data files are available in the online version of the paper.

\section{ACKNOWLEDGMENTS}

We thank Armed Forces Research Institute of Medical Sciences, Thailand for Vero cells, C6/36 cells, mAb 4G2 to DENV envelope protein, and viruses DENV1, DENV-2 and DENV-3; and M. Nguyen-De Bernon for technical assistance. Supported by the Medical Research Council, UK, the Wellcome Trust, UK (G.R.S.), the National Institute for Health Research Biomedical Research Centre, Funding Scheme, European Commission Seventh Framework Programme (FP7/20072013) for the DENFREE project (under grant agreement 282378 ), Laboratory of Excellence Integrative Biology of Emerging Infectious Diseases (ANR-10-LABX62-IBEID to F.A.R.), Agence Nationale de la Recherche (FlaviStem/I1378 to F.A.R.) and the Thailand Research Fund through the Royal Golden Jubilee PhD (P.S. and J.M.).

\section{AUTHOR CONTRIBUTIONS}

W.D., P.S., W.W., A.R., G.B.-S. and T.D. performed experiments; A.S. and V.-M.C.-L. provided ZIKV strains HD78788 and PF13; P.M. provided specimens; F.A.R., J.M. and G.R.S. conceived of the experiments; and W.D., J.M. and G.R.S. analyzed data and wrote the paper

\section{COMPETING FINANCIAL INTERESTS}

The authors declare competing financial interests: details are available in the online version of the paper.

Reprints and permissions information is available online at http://www.nature.com/ reprints/index.html.

1. Musso, D. \& Gubler, D.J. Zika Virus. Clin. Microbiol. Rev. 29, 487-524 (2016).

2. Lazear, H.M. \& Diamond, M.S. Zika virus: new clinical syndromes and its emergence in the western hemisphere. J. Virol. 90, 4864-4875 (2016). 
3. World Health Organization. Zika Virus Fact sheet. http://www.who.int/mediacentre/ factsheets/zika/en/ (2016)

4. Duffy, M.R. et al. Zika virus outbreak on Yap Island, Federated States of Micronesia. N. Engl. J. Med. 360, 2536-2543 (2009).

5. Cao-Lormeau, V.M. et al. Zika virus, French Polynesia, South Pacific, 2013. Emerg. Infect. Dis. 20, 1085-1086 (2014).

6. Zanluca, C. et al. First report of autochthonous transmission of Zika virus in Brazil. Mem. Inst. Oswaldo Cruz 110, 569-572 (2015).

7. Campos, G.S., Bandeira, A.C. \& Sardi, S.I. Zika virus outbreak, Bahia, Brazil. Emerg. Infect. Dis. 21, 1885-1886 (2015).

8. Cao-Lormeau, V.M. et al. Guillain-Barré Syndrome outbreak associated with Zika virus infection in French Polynesia: a case-control study. Lancet 387, 1531-1539 (2016).

9. Teixeira, M.G., da Conceição N Costa, M., de Oliveira, W.K., Nunes, M.L. \& Rodrigues, L.C. The epidemic of Zika virus-related microcephaly in Brazil: detection, control, etiology, and future scenarios. Am. J. Public Health 106, 601-605 (2016).

10. Mlakar, J. et al. Zika virus associated with microcephaly. N. Engl. J. Med. 374, 951958 (2016).

11. World Health Organization. Zika emergency. http://www.who.int/mediacentre/news/ statements/2016/1st-emergency-committee-zika/en/ (2016).

12. Musso, D., Cao-Lormeau, V.M. \& Gubler, D.J. Zika virus: following the path of dengue and chikungunya? Lancet 386, 243-244 (2015).

13. Cardoso, C.W. et al. Outbreak of exanthematous illness associated with Zika, chikungunya, and dengue viruses, Salvador, Brazil. Emerg. Infect. Dis. 21, 2274-2276 (2015).

14. Teixeira, M.G. et al. East/Central/South African genotype chikungunya virus, Brazil, 2014. Emerg. Infect. Dis. 21, 906-907 (2015).

15. Weaver, S.C. \& Lecuit, M. Chikungunya virus and the global spread of a mosquito-borne disease. N. Engl. J. Med. 372, 1231-1239 (2015).

16. Guzman, M.G. \& Harris, E. Dengue. Lancet 385, 453-465 (2015).

17. Morrison, T.E. Reemergence of chikungunya virus. J. Virol. 88, 11644-11647 (2014).

18. Brathwaite Dick, O. et al. The history of dengue outbreaks in the Americas. Am. J. Trop. Med. Hyg. 87, 584-593 (2012)

19. Castanha, P.M. et al. Force of infection of dengue serotypes in a population-based study in the northeast of Brazil. Epidemiol. Infect. 141, 1080-1088 (2013).

20. Braga, C. et al. Seroprevalence and risk factors for dengue infection in socio-economcally distinct areas of Recife, Brazil. Acta Trop. 113, 234-240 (2010).

21. Screaton, G., Mongkolsapaya, J., Yacoub, S. \& Roberts, C. New insights into the immunopathology and control of dengue virus infection. Nat. Rev. Immunol. 15, 745-759 (2015).

22. Buathong, R. et al. Detection of Zika virus infection in Thailand, 2012-2014. Am. J. Trop. Med. Hyg. 93, 380-383 (2015)

23. Lanciotti, R.S. et al. Genetic and serologic properties of Zika virus associated with an epidemic, Yap State, Micronesia, 2007. Emerg. Infect. Dis. 14, 1232-1239 (2008).

24. Guzman, M.G. et al. Neutralizing antibodies after infection with dengue 1 virus. Emerg. Infect. Dis. 13, 282-286 (2007).

25. Sabin, A.B. Research on dengue during World War II. Am. J. Trop. Med. Hyg. 1, 30-50 (1952).

26. Guzman, M.G. et al. Dengue: a continuing global threat. Nat. Rev. Microbiol. 8 Suppl, S7-S16 (2010).
27. Dejnirattisai, W. et al. A new class of highly potent, broadly neutralizing antibodies isolated from viremic patients infected with dengue virus. Nat. Immunol. 16, 170-177 (2015).

28. Halstead, S.B. \& O'Rourke, E.J. Dengue viruses and mononuclear phagocytes. I. Infection enhancement by non-neutralizing antibody. J. Exp. Med. 146, 201-217 (1977).

29. Halstead, S.B. \& O'Rourke, E.J. Antibody-enhanced dengue virus infection in primate leukocytes. Nature 265, 739-741 (1977).

30. Zompi, S. \& Harris, E. Animal models of dengue virus infection. Viruses 4, 62-82 (2012).

31. Brandt, W.E., McCown, J.M., Gentry, M.K. \& Russell, P.K. Infection enhancement of dengue type 2 virus in the U-937 human monocyte cell line by antibodies to flavivirus cross-reactive determinants. Infect. Immun. 36, 1036-1041 (1982).

32. Rouvinski, A. et al. Recognition determinants of broadly neutralizing human antibodies against dengue viruses. Nature 520, 109-113 (2015)

33. Barba-Spaeth, G. et al. Structural basis of potent antibody cross-neutralization between Zika and Dengue viruses. Nature (in the press).

34. Calvet, G. et al. Detection and sequencing of Zika virus from amniotic fluid of fetuses with microcephaly in Brazil: a case study. Lancet Infect. Dis.16, 653-660 (2016).

35. Martines, R.B. et al. Notes from the Field: Evidence of Zika virus infection in brain and placental tissues from two congenitally infected newborns and two fetal losses - Brazil, 2015. MMWR Morb. Mortal. Wkly. Rep. 65, 159-160 (2016).

36. Meaney-Delman, D. et al. Zika virus infection among U.S. pregnant travelers - August 2015-February 2016. MMWR Morb. Mortal. Wkly. Rep. 65, 211-214 (2016).

37. Sarno, M. et al. Zika virus infection and stillbirths: a case of hydrops fetalis, hydranencephaly and fetal demise. PLoS Negl. Trop. Dis. 10, e0004517 (2016).

38. Cugola, F.R. et al. The Brazilian Zika virus strain causes birth defects in experimental models. Nature 534, 267-271 (2016).

39. Miner, J.J. et al. Zika virus infection during pregnancy in mice causes placental damage and fetal demise. Cell 165, 1081-1091 (2016).

40. Li, C. et al. Zika virus disrupts neural progenitor development and leads to microcephaly in mice. Cell Stem Cell 19, 1-7 (2016).

41. Dang, J. et al. Zika virus depletes neural progenitors in human cerebral organoids through activation of the innate immune receptor TLR3. Cell Stem Cell doi:10.1016/j. stem.2016.04.014 (6 May 2016).

42. Garcez, P.P. et al. Zika virus impairs growth in human neurospheres and brain organoids Science 352, 816-818 (2016).

43. Brasil, P. et al. Zika virus infection in pregnant women in Rio de Janeiro - preliminary report. N. Engl. J. Med. doi:10.1056/NEJMoa1602412 (4 March 2016).

44. Cauchemez, S. et al. Association between Zika virus and microcephaly in French Polynesia, 2013-15: a retrospective study. Lancet 387, 2125-2132 (2016).

45. Stiasny, K., Kiermayr, S., Holzmann, H. \& Heinz, F.X. Cryptic properties of a cluster of dominant flavivirus cross-reactive antigenic sites. J. Virol. 80, 9557-9568 (2006).

46. Paul, L.M. et al. Dengue virus antibodies enhance Zika virus infection. bioRxiv $10.1101 / 050112$ (2016).

47. World Health Organization (WHO) Strategic Advisory Group of Experts. Dengue vac cine. http://www.who.int/immunization/sage/meetings/2016/april/SAGE_April_2016 Meeting Web summary.pdf?ua=1 (2016). 


\section{ONLINE METHODS}

Samples. Blood samples were collected after written informed consent was obtained, with approval of the ethical committee of the Khon Kaen and Siriraj Hospitals in Thailand and the Riverside Ethics Committee in UK. The serotypes of DENV infection was determined by RT-PCR detection of the viral genome ${ }^{48}$. Samples were collected around 6 months after recovery from dengue illness (Table 1). Secondary DENV infection was classified based on the ratio of $<1.8$, anti-DENV IgM to anti-DENV IgG, measured by IgM and IgG capture ELISA ${ }^{48}$. The dengue disease severity was classified according to the WHO 1997 classification. Pooled serum from convalescent subjects was obtained by combining serum taken from dengue-infected patients around 1-2 weeks after recovery. Plasma pooled from control subjects not infected with DENV was obtained from normal healthy subjects that had no detectable anti-DENV antibodies.

Cells, reagents and antibodies. Vero cells (a gift from Armed Forces Research Institute of Medical Sciences (AFRIMS)), 293T cells and U937 cells were cultured at $37^{\circ} \mathrm{C}$ in MEM, DMEM and RPMI-1640, respectively. C6/36 cells (a gift from AFRIMS) were grown in Leibovitz L- 15 at $28^{\circ} \mathrm{C}$. All medium contained $10 \%$ heat-inactivated fetal bovine serum, 100 units $/ \mathrm{ml}$ penicillin and $100 \mathrm{mg} / \mathrm{ml}$ streptomycin. All cell lines were free from mycoplasma contamination.

Alkaline phosphatase (ALP)-conjugated anti-human IgG (A9544) and horseradish-peroxidase-conjugated anti-human IgG (P0214) were purchased from Sigma and Dako, respectively. Mouse mAb 4G2 to DENV envelope protein was a gift from AFRIMS. RPMI-1640 (R8758), DMEM (D5046), p-nitrophenylphosphate (PNPP, N2770-50), Bovine serum albumin (BSA, A7030), diaminobenzidine (D5905), and polyethylenimine (408727; Sigma) were from Sigma. MEM (31095) and Leibovitz L-15 (11415) were from Gibco and UltraDOMA-PF (12727F) was from Lonza.

Viral stocks. All viruses were grown in C6/36 cells. A.S. provided ZIKV strain HD78788 (African strain). ZIKV strain PF13/251013-18 (PF13) was isolated from a patient during the ZIKV outbreak in French Polynesia in 2013. DENV-1 (Hawaii), DENV-2 (16681), and DENV-3 (H87) were gifts from AFRIMS. DENV-4 (1-0093) was isolated from a DENV-4-infected patient. Viruscontaining supernatants were clarified by centrifugation at 2,000 r.p.m. at $4{ }^{\circ} \mathrm{C}$ before being stored at $-80^{\circ} \mathrm{C}$. Viral titers were determined by a focus-forming assays on Vero cells ${ }^{27}$. All viral stocks were free from mycoplasma contamination.

Expression of human mAbs to DENV envelope protein. A pair of plasmids containing heavy and light chains of IgG1 were co-transfected into 293T cells using the polyethylenimine method and cultured in protein-free media. Culture supernatant containing antibodies was harvested after $5 \mathrm{~d}$.

Determination of ZIKV cross-reactivity of anti-DENV antibodies by ELISA. A MAXISORP immunoplate (442404; NUNC) was coated with $50 \mu \mathrm{l} \mathrm{of} 5 \mu \mathrm{g} / \mathrm{ml}$ of mouse antibody $4 \mathrm{G} 2$ to envelope protein (a mouse antibody to the fusion loop that cross-reacts with ZIKV). Plates were blocked with 3\% BSA (A7030, Sigma) for $1 \mathrm{~h}$, followed by incubation with viral supernatant. After $1 \mathrm{~h}, 10 \mathrm{mg} / \mathrm{ml} \mathrm{human}$ $\mathrm{mAbs}$ to DENV or serially diluted plasma was added. The reaction was visualized by ALP-conjugated anti-human IgG antibody at a 1:10,000 dilution (A9544; Sigma) and PNPP substrate (N2770, Sigma). Reactions were stopped with $\mathrm{NaOH}$ and the absorbance was measured at $405 \mathrm{~nm}$. Endpoint titers (EPTs) were defined as reciprocal plasma dilutions that corresponded to two times the average OD values obtained with mock antigen.

Neutralization assay. The focus reduction neutralization assay (FRNT) was employed to determine the neutralizing potential of antibodies. Virus was incubated with serial dilutions of antibodies or plasma samples for $1 \mathrm{~h}$ at $37^{\circ} \mathrm{C}$. The mixtures were then added to Vero cells and incubated for $2 \mathrm{~d}$ (for ZIKV) or $3 \mathrm{~d}$ (for DENV). Focus-forming assays were then performed as described ${ }^{27}$. Vero cells were stained with mAb 4G2 (to envelope protein; cell culture supernatant (a gift from AFRIMS)), followed by peroxidase-conjugated goat anti-mouse immunoglobulin at a 1:1,000 dilution (P0047; Sigma). The foci (infected cells) were visualized by adding the peroxidase substrate DAB (D5905, Sigma). The percentage focus reduction was calculated and 50\% FRNT was calculated using the probit program from the SPSS package.

Antibody-dependent enhancement assay. Serially diluted $\mathrm{mAb}$ or plasma samples were incubated with virus at moi of 2 (ZIKV) or 5 (DENV) for $1 \mathrm{~h}$ at 37 ${ }^{\circ} \mathrm{C}$ before adding to U937 cells. After incubation for $2 \mathrm{~d}$ (ZIKV) or $3 \mathrm{~d}$ (DENV), supernatants were harvested and viral titers determined by focus forming assays. Fold enhancement was calculated by comparison to viral titers in the presence or absence of antibody. For flow cytometry, infected U937 cells were fixed and permeabilized in $3.7 \%$ formaldehyde and $0.5 \%$ saponin, respectively. Intracellular staining was performed with $4 \mathrm{G} 2$ conjugated with Alexa Fluor 647 at $4{ }^{\circ} \mathrm{C}$ for 30 min. Infected cells were analyzed by BD FACSVerse.

ADE inhibition by human $\mathrm{mAbs}$ was performed by premixing pooled convalescent dengue hyper-immune serum at 1:10,000 dilution (a peak enhancing dilution) with serially diluted $\mathrm{mAb}$ before performing the $\mathrm{ADE}$ assay as described above.

48. Mongkolsapaya, J. et al. Original antigenic sin and apoptosis in the pathogenesis of dengue hemorrhagic fever. Nat. Med. 9, 921-927 (2003) 